04

\title{
Резистивное переключение мемристоров на основе стабилизированного диоксида циркония сложными сигналами
}

\author{
() Д.О. Филатов, Д.А. Антонов, И.Н. Антонов, А.И. Белов, В.Н. Баранова, М.Е. Шенина, О.Н. Горшков \\ Нижегородский государственный университет им. Н.И. Лобачевского, \\ Нижний Новгород, Россия \\ E-mail: dmitry_filatov@inbox.ru
}

Поступила в Редакцию 31 октября 2019 г.

В окончательной редакции 31 октября 2019 г.

Принята к публикации 19 октября 2019 г.

\begin{abstract}
Изучены особенности резистивного переключения в экспериментальных образцах мемристоров на основе тонких пленок стабилизированного иттрием диоксида циркония треугольными импульсами, на которые накладывался высокочастотный синусоидальный сигнал. Обнаружено уменьшение значений напряжения переключения мемристора из низкоомного состояния в высокоомное и обратно, а также увеличение отношения значений силы тока через мемристор в указанных состояниях и долговременной стабильности тока при наложении синусоидального сигнала на переключающие импульсы по сравнению с переключениями треугольными импульсами без синусоидального сигнала. Улучшение характеристик резистивного переключения связано с резонансной активацией миграции ионов кислорода по вакансиям в переменном внешнем электрическом поле.
\end{abstract}

Ключевые слова: мемристор, резистивное переключение, стабилизированный диоксид циркония, резонансная активация.

DOI: 10.21883/FTT.2020.04.49120.620

\section{1. Введение}

Эффект резистивного переключения (РП) заключается в обратимом бистабильном (мультистабильном) изменении электросопротивления диэлектрической прослойки между двумя проводящими электродами под действием электрического напряжения, приложенного к электродам $[1,2]$. Общепринятым в настоящее время механизмом РП в оксидах металлов считается разрушение и восстановление проводящих каналов (филаментов) из вакансий кислорода, формирующихся внутри слоя функционального диэлектрика под действием электрического поля между электродами в ходе предварительного электроформинга [3]. Электронные приборы, принцип действия которых основан на эффекте РП, получили название мемристоров [4]. Данные приборы считаются перспективными для создания устройств энергонезависимой компьютерной памяти нового поколения [5], нейроморфных вычислительных устройств [6] и др.

В настоящий момент широкое практическое применение мемристоров сдерживается недостаточной стабильностью параметров РП, таких как напряжение переключения пленок функционального диэлектрика из состояния с высоким сопротивлением (СВC) в состояние с низким сопротивлением (CHC) - так называемый SET процесс - и обратно (RESET процесс) $V_{\text {SET }}$ и $V_{\text {RESET }}$ соответственно, значения силы тока через мемристор в СВC и $\mathrm{CHC} I_{\mathrm{OFF}}$ и $I_{\mathrm{ON}}$ соответственно, а также деградацией мемристоров в процессе эксплуатации [7]. Разрушение и восстановление филаментов носят стохастический характер, поскольку типичные поперечные размеры филаментов составляют $\sim 1-10 \mathrm{~nm}[8]$, а в переключениях участвует лишь ограниченное (малое) количество ионов кислорода (кислородных вакансий) вблизи оконечности филамента, ближайшей к интерфейсу с электродом [9]. В настоящее время развивается ряд различных подходов к повышению стабильности параметров мемристоров: выбор оптимальных комбинаций материалов диэлектрика и электродов [10], использование многослойных диэлектриков [11], применение переключающих сигналов специальной формы $[12,13]$ и др. Несмотря на значительный прогресс в области повышения долговечности мемристоров, достигнутый в последние годы (так, в экспериментальных образцах было достигнуто число переключений до $\left.10^{9}[11]\right)$, проблема долговременной нестабильности параметров мемристоров еще далека от окончательного решения.

В [14] был применен инновационный подход к решению проблемы повышения стабильности мемристоров, основанный на использовании эффекта конструктивной роли шума в нелинейных системах. Для этого на прямоугольный переключающий сигнал накладывался белый шум регулируемой мощности. Было отмечено повышение временно́й стабильности мемристора в случае переключения с наложением шума, при этом зависимость стабильности РП от мощности шума имела выраженный максимум. В [15] был использован подход к повышению стабильности параметров мемристоров, основанный на концепции стохастического резонанса [16]. Было экспериментально продемонстрировано улучшение соотношения сопротивлений исследуемых мемристоров в СВС и СНС в условиях стохастического резонанса (до 3.4 раза), 
при этом разброс параметров мемристоров от одного устройства к другому в пределах $60 \%$ слабо влиял на обнаруженный эффект.

В [17] исследовано РП в контакте проводящего зонда атомно-силового микроскопа (АСM) к тонкой пленке стабилизированного иттрием диоксида циркония $\mathrm{ZrO}_{2}(\mathrm{Y})$ на проводящей подложке при переключении треугольными импульсами с наложением высокочастотного (ВЧ) синусоидального сигнала. $\mathrm{ZrO}_{2}(\mathrm{Y})$ считается перспективным функциональным диэлектриком для мемристивных приборов в связи с высокой подвижностью кислородных вакансий [18]. Одной из проблем в разработке мемристивных устройств энергонезависимой памяти является проблема масштабирования [19]: закономерности РП, установленные при исследованиях модельных мемристоров с микрометровыми размерами электродов (под которыми может формироваться большое количество филаментов), оказываются отличными от закономерностей РП мемристоров с электродами нанометровых размеров, под которыми может поместиться небольшое количество филаментов (в пределе - один филамент). Типичные размеры области контакта острия ACM зонда с Pt покрытием к поверхности пленок $\mathrm{ZrO}_{2}(\mathrm{Y}) D_{p}<10 \mathrm{~nm}$ [20], что соответствует ожидаемым размерам ячеек перспективной мемристивной памяти [21]. Таким образом, контакт проводящего АСМ зонда к поверхности диэлектрической пленки на проводящей подложке представлял собой удобную модельную систему (виртуальный мемристор) для изучения особенностей РП в масштабах, соответствующих ожидаемым размерам перспективных мемристивных устройств [22]. В [17] было обнаружено, что отношение значений силы тока через контакт АCM зонда к поверхности пленки $\mathrm{ZrO}_{2}(\mathrm{Y})$ в $\mathrm{CHC}$ и $\mathrm{CBC} I_{\mathrm{ON}} / I_{\mathrm{OFF}}$, а также их временна́я стабильность увеличиваются при наложении на треугольные переключающие импульсы ВЧ синусоидального сигнала частотой $f \sim 1 \mathrm{kHz}$ по сравнению с переключениями треугольными импульсами без наложения синусоидального сигнала. Обнаруженный эффект был связан с резонансной активацией миграции ионов кислорода по вакансиям в переменном внешнем электрическом поле.

В настоящей работе исследовано РП в макетах мемристивных устройств памяти на базе тонких пленок $\mathrm{ZrO}_{2}(\mathrm{Y})$ при переключении треугольными импульсами с наложением ВЧ синусоидального сигнала. Целью настоящей работы являлось исследование влияния ВЧ сигнала на РП мемристорных устройств с макроскопическими (микрометровых размеров) электродами в сравнении с результатами, полученными ранее при исследовании РП в нанометровом масштабе с помощью АCM [17].

\section{2. Методика эксперимента}

Исследуемые структуры $\mathrm{Au} / \mathrm{Zr} / \mathrm{ZrO}_{2}(\mathrm{Y})$ формировались на $\mathrm{Si}(001)$ подложках с подслоями металлизации Тi и TiN (толщиной $25 \mathrm{~nm}$ каждый), следующим образом:

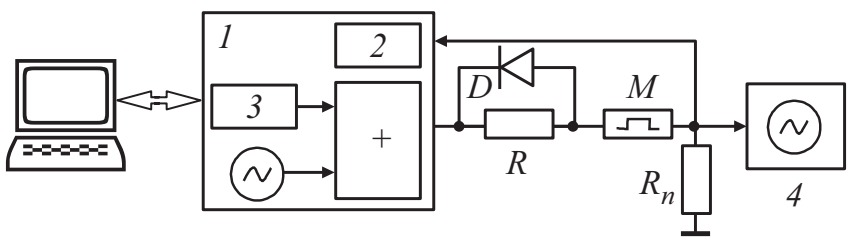

Рис. 1. Схема эксперимента по исследованию РП мемристора треугольными импульсами с наложением ВЧ синусоидального сигнала: 1 - АCM контроллер Solver Pro, 2 - АЦП, 3 - ЦАП, 4 - осциллограф, $M$ - исследуемый мемристор, $R$ - ограничивающий резистор, $D-$ диод, $R_{n}-$ сопротивление нагрузки.

методом ВЧ магнетронного осаждения при температуре подложки $T_{g}=300^{\circ} \mathrm{C}$ наносились слои $\mathrm{ZrO}_{2}(\mathrm{Y})$ $\left(\sim 12 \mathrm{~mol} . \% \mathrm{Y}_{2} \mathrm{O}_{3}\right)$ толщиной $40 \mathrm{~nm}$, поверх которых методом магнетронного осаждения на постоянном токе при $T_{g}=200^{\circ} \mathrm{C}$ наносились слои $\mathrm{Zr}(8 \mathrm{~nm})$ и $\mathrm{Au}(40 \mathrm{~nm})$. На основе данных структур методом фотолитографии формировались макеты мемристорных устройств памяти типа кросс-пойнт с размером активной области $20 \times 20 \mu \mathrm{m}$. Активные области мемристоров закрывались защитным слоем $\mathrm{SiO}_{2}$. Более детально процедура формирования мемристорных структур, а также результаты исследований их структуры и электрофизических свойств макетов мемристорных устройств на их основе приведены в [23].

Исследования РП проводились в схеме 1M1R (1 мемристор - 1 резистор) при помощи установки, схема которой приведена на рис. 1. В качестве программируемого источника прикладываемого к электродам мемристора напряжения, зависящего от времени, $V(t)$, использовался цифро-аналоговый преобразователь (ЦАП) контроллера АCM Solver Pro производства компании НТ-МДТ (Россия) под управлением программного обеспечения Nova 1138 (разработчик - компания НТ-МДТ) при помощи оболочки для разработки пользовательских скриптов Nova Power Script. Ограничение силы тока через мемристор I в СНС осуществлялось при помощи ограничивающего резистора $R=10 \mathrm{k} \Omega$, шунтированного кремниевым диодом Д220. Сигнал, пропорциональный силе тока через мемристор, $I(t)$, снимался с сопротивления нагрузки $R_{n}=1 \mathrm{k} \Omega \sim 0.1 R$ и подавался на аналого-цифровой преобразователь (АЦП) контроллера ACM Solver Pro. Контроль формы осциллограмм тока через мемристор $I(t)$ осуществлялся при помощи цифрового осциллографа Agilent 3000A.

В эксперименте измерялись циклические вольт-амперные характеристики (BAX) мемристора $I(V)$ при линейной развертке $V$ от $V_{\min }<V_{\mathrm{RESET}}$ до $V_{\max }>V_{\mathrm{SET}}$ и обратно до $V_{\min }$ и т.д. Значения $V_{\min }$ и $V_{\max }$ варьировались в пределах $-(3-5) \mathrm{V}$ и $+(5-6) \mathrm{V}$ соответственно. Период повторения переключающих импульсов $T_{i}$ составлял $1-5 \mathrm{~s}$.

Из анализа циклических ВАХ определялись такие параметры РП, как $V_{\mathrm{SET}}$ и $V_{\text {RESET, a также значе- }}$ 


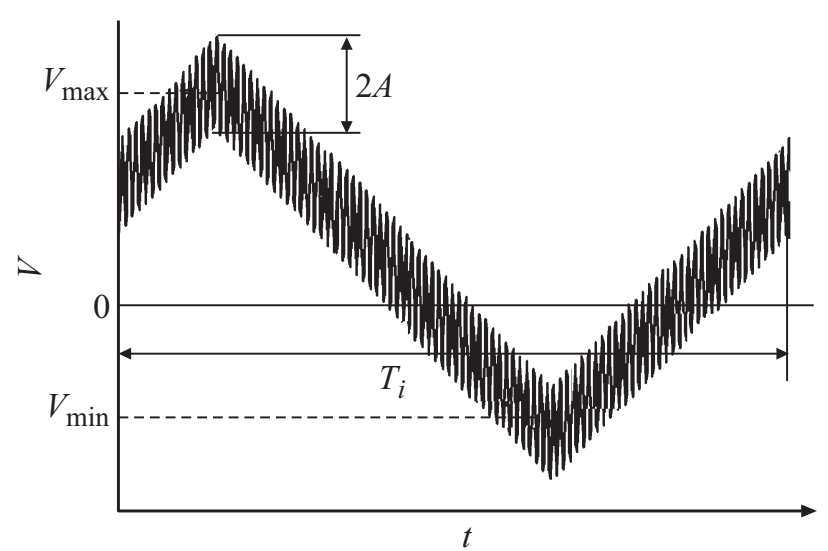

Рис. 2. Осциллограмма переключающего сигнала при наложении ВЧ синусоидального сигнала (качественно).

ния $I$ в СВС и СНC $I_{\mathrm{OFF}}$ и $I_{\mathrm{ON}}$ соответственно, при $0<V_{g}=V_{\text {read }}<V_{\mathrm{SET}}$.

С целью исследования влияния дополнительного ВЧ синусоидального сигнала на параметры РП, на треугольные переключающие импульсы накладывалось переменное синусоидальное напряжение с амплитудой $A=0-2 \mathrm{~V}$ и частотой $f=0.4-10 \mathrm{kHz}$. Пример осциллограммы переключающего пилообразного напряжения $V(t)$ с наложенным синусоидальным сигналом приведен на рис. 2. В качестве источника синусоидального напряжения использовался программируемый цифровой генератор синусоидального сигнала контроллера АСМ Solver Pro. Выбор частоты синусоидального сигнала $f$ был обусловлен следующими соображениями. Как уже упоминалось выше, (см. [17]) улучшение параметров РП при переключении пилообразным напряжением с наложением ВЧ синусоидального сигнала, наблюдавшееся ранее с использованием АСМ, было связано c резонансной активацией миграции ионов $\mathrm{O}^{2-}$ по вакансиям кислорода в $\mathrm{ZrO}_{2}(\mathrm{Y})$ под действием переменного внешнего напряжения. С другой стороны, как было установлено в [24,25], энергия активации миграции ионов $\mathrm{O}^{2-}$ по вакансиям кислорода, которая является основным фундаментальным элементарным процессом механизма РП, $E_{a}=0.52-0.68 \mathrm{~V}$ (для так называемых активных вакансий, имеющих атом Y в первой координационной сфере [26]). Исходя из указанных значений $E_{a}$, характерная частота перескоков ионов $\mathrm{O}^{2-}$ на соседние вакансии $f_{i}$ может быть оценена по формуле

$$
f_{i} \sim f_{0} \exp \left(-\frac{E_{a}}{k T}\right),
$$

где $f_{0}-$ фононная частота $\left(\sim 10^{13} \mathrm{~Hz}\right), k-$ постоянная Больцмана, $T$ - абсолютная температура. Согласно (1), для значений $E_{a}$, приводимых в $[24,25]$, значения $f_{i}$ при $T=300 \mathrm{~K}$ лежат в диапазоне $0.4-8 \mathrm{kHz}$. Значения частоты ВЧ синусоидального сигнала $f$ в экспериментах, проводимых в рамках настоящей работы, выбирались в пределах указанного диапазона.
Параметры процесса РП в мемристоре при переключении треугольными импульсами с наложением ВЧ синусоидального сигнала (рис. 2) $(A=0.2 \mathrm{~V}, f=1 \mathrm{kHz})$ и без него

\begin{tabular}{c|c|c|c|c}
\hline \multirow{2}{*}{ Параметр } & \multicolumn{2}{|c|}{$\begin{array}{c}\text { С наложением ВЧ } \\
\text { синусоидального сигнала }\end{array}$} & \multicolumn{2}{c}{ Без наложения } \\
\cline { 2 - 5 } & среднее & $\sigma$ & среднее & $\sigma$ \\
\hline$V_{\mathrm{SET}}, \mathrm{V}$ & 3.348 & 0.828 & 4.782 & 0.133 \\
$V_{\mathrm{RESET}}, \mathrm{V}$ & -2.501 & 0.378 & -3.446 & 0.340 \\
$I_{\mathrm{ON}}, \mathrm{mA}$ & 0.10 & 0.043 & 0.18 & 0.19 \\
$I_{\mathrm{OFF}}, \mu \mathrm{A}$ & 2 & 2 & 7 & 9 \\
$I_{\mathrm{ON}} / I_{\mathrm{OFF}}$ & 50 & 28.5 & 25.7 & 5.9
\end{tabular}

\section{3. Результаты и обсуждение}

На рис. 3 приведен пример циклической ВАХ мемристора, на которой наблюдается выраженный гистерезис, обусловленный биполярным РП в пленке $\mathrm{ZrO}_{2}(\mathrm{Y})$. Заметим, что ВАХ исследованных мемристоров является асимметричной: значения $\left|V_{\mathrm{SET}}\right|>\left|V_{\mathrm{RESET}}\right|$. Заметим также, что переход из CBC в CHC (SET процесс) является резким, в то время как обратный переход из СHC в CBC (RESET процесс) является более плавным (такой тип RESET процесса в англоязычной литературе получил название gradual reset). Подобная форма BAX является типичной для мемристоров с функциональными диэлектриками на базе оксидов металлов, принцип действия которых основан на процессах окисления и восстановления атомов металлов вблизи интерфейсов функционального диэлектрика с металлическими электродами (так называемые redox процессы) $[1]$.

На рис. 4 приведены зависимости значений $I_{\mathrm{ON}}$ и $I_{\mathrm{OFF}}$ при $V_{\text {read }}=2 \mathrm{~V}$ от количества циклов переключения $N$ для случаев переключения треугольными импульсами с наложением синусоидального сигнала с амплитудой $A=0.2 \mathrm{~V}$ и частотой $f=1 \mathrm{kHz}$ и без наложения сину-

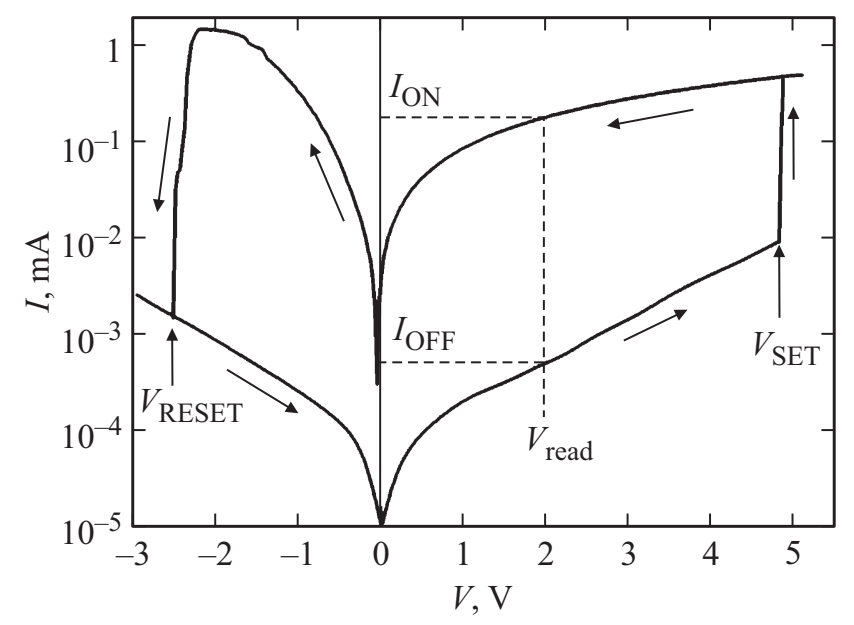

Рис. 3. Циклическая BAX мемристора на базе структуры $\mathrm{Au} / \mathrm{Zr} / \mathrm{ZrO}_{2}(\mathrm{Y}) / \mathrm{TiN} / \mathrm{Ti} / \mathrm{SiO}_{2} / \mathrm{Si}$. 


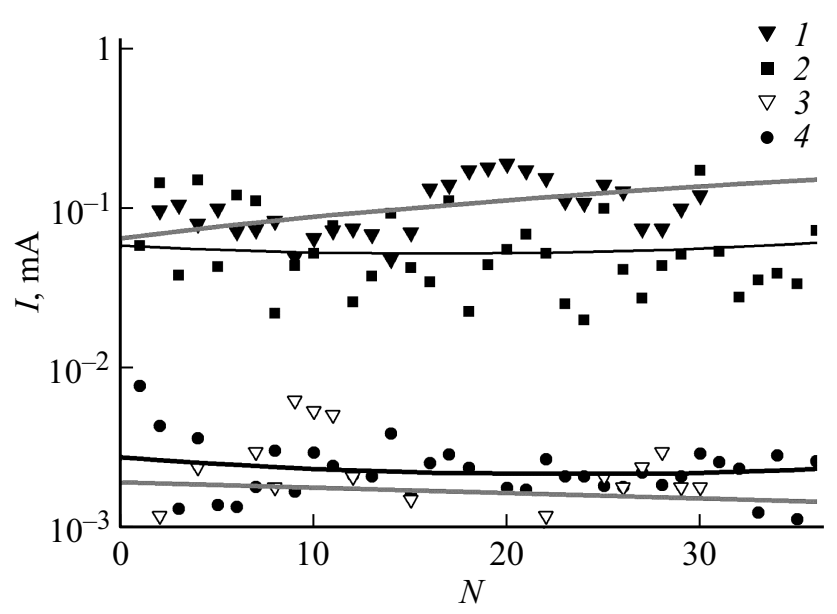

Рис. 4. Зависимости силы тока через мемристор в СНС $I_{\mathrm{ON}}(1,2)$ и СВC $I_{\mathrm{OFF}}(3,4)$ от количества переключений $N$. $V_{\text {read }}=2 \mathrm{~V}$. $1,3-\mathrm{c}$ наложением синусоидального сигнала $(A=0.2 \mathrm{~V}, f=1 \mathrm{kHz}), 2,4-$ без наложения синусоидального сигнала.

соидального сигнала. В таблице приведены усредненные параметры РП мемристора $\left(V_{\mathrm{SET}}, V_{\mathrm{RESET}}, I_{\mathrm{ON}}\right.$ и $I_{\mathrm{OFF}}$, а также отношения $\left.I_{\mathrm{ON}} / I_{\mathrm{OFF}}\right)$, определенные из анализа серии из 35 циклических ВАХ.
Значение отношения $I_{\mathrm{ON}} / I_{\mathrm{OFF}}$ при переключении с наложением ВЧ синусоидального сигнала существенно (примерно 2 раза) превышало соответствующее значение при переключении треугольными импульсами без наложения синусоидального сигнала (см. таблицу). Аналогичное увеличение отношения $I_{\mathrm{ON}} / I_{\mathrm{OFF}}$ при наложении ВЧ синусоидального сигнала наблюдалось ранее при исследовании локального РП в пленках $\mathrm{ZrO}_{2}(\mathrm{Y})$ при помощи АСМ [17].

Также на рис. 4 показаны линии тренда, аппроксимирующие зависимости $I_{\mathrm{ON}}$ и $I_{\mathrm{OFF}}$ от $N$ полиномом 2-го порядка. В случае переключения треугольными импульсами с наложением ВЧ синусоидального сигнала линии тренда соответствуют возрастанию $I_{\mathrm{ON}}$ и $I_{\mathrm{OFF}} \mathrm{c}$ увеличением $N$. В случае переключения треугольными импульсами без наложения ВЧ синусоидального сигнала линии тренда как для $I_{\mathrm{ON}}$, так и для $I_{\mathrm{OFF}}$ остаются практически постоянными при изменении $N$ в пределах от 1 до 35.

В [17] для результатов, полученных методом АСМ, было отмечено противоположное поведение трендов $I_{\mathrm{ON}}$ и $I_{\mathrm{OFF}}$.

На рис. 5, $a$ и $c$ представлены гистограммы распределений $V_{\mathrm{SET}}$, а на рис. $5, b$ и $d-V_{\mathrm{RESET}}$ для случаев переключения с наложением ВЧ синусоидального сигнала (рис. $5, a$ и $b$ ) и без (рис. $5, c$ и $d$ ). В обоих случаях
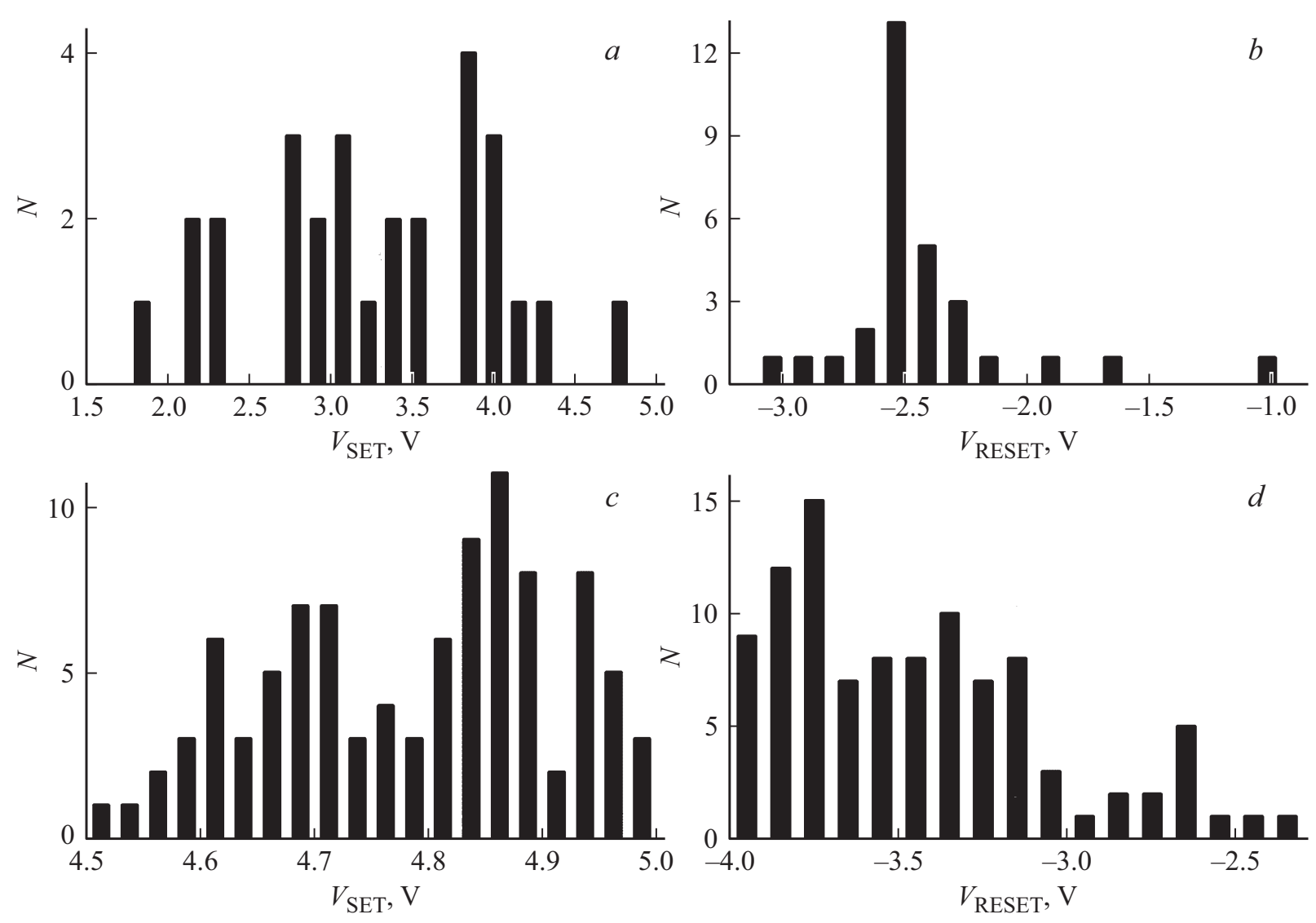

Рис. 5. Гистограммы распределений напряжений переключения мемристора из $\mathrm{CBC}$ в $\mathrm{CHC} V_{\mathrm{SET}}(a, c)$ и обратно из CHC в $\mathrm{CBC}$ $V_{\mathrm{RESET}}(b, d)$ с наложением синусоидального сигнала $(A=0.2 \mathrm{~V}, f=1 \mathrm{kHz})(a, b)$ и без наложения синусоидального сигнала $(c, d)$. 
(как в случае наложения ВЧ синусоидального сигнала, так и без него) распределения $V_{\mathrm{SET}}$ и $V_{\mathrm{RESET}}$ были существенно асимметричными. В случае переключения с наложением ВЧ синусоидального сигнала значения разброса значений как $V_{\mathrm{SET}}$, так и $V_{\mathrm{RESET}}$ от одного цикла переключения к другому были значительно больше, чем соответствующие значения при переключении треугольными импульсами без наложения синусоидального сигнала (см. таблицу). Отметим, что в [17] при изучении влияния наложения ВЧ синусоидального сигнала на треугольные переключающие импульсы методом АСМ наблюдалась обратная ситуация: распределения $V_{\mathrm{SET}}$ и $V_{\mathrm{RESET}}$ в случае переключения с наложением синусоидального сигнала были более узкими, чем при переключении треугольными импульсами.

С другой стороны, относительный разброс значений $I_{\mathrm{ON}}$ от цикла к циклу $\sigma I_{\mathrm{ON}} /\left\langle I_{\mathrm{ON}}\right\rangle$, где $\left\langle I_{\mathrm{ON}}\right\rangle-$ среднее значение $I_{\mathrm{ON}}$ в серии измерений, $\sigma I_{\mathrm{ON}}-$ среднеквадратичное отклонение значений $I_{\mathrm{ON}}$ от $\left\langle I_{\mathrm{ON}}\right\rangle$, при измерениях с наложением синусоидального сигнала был в $\approx 2$ раза меньше, чем без синусоидального сигнала $(\approx 0.43$ и $\approx 1.06$ соответственно). В то же время, в измерениях методом АСМ, [17] относительная дисперсия значений $I_{\mathrm{ON}}$ и $I_{\mathrm{OFF}}$ была приблизительно одинаковой при переключении с наложением синусоидального сигнала и без него. Сравнение дисперсии значений $I_{\mathrm{OFF}}$ затрудняется малыми значениями $I_{\mathrm{OFF}}$, измеряемыми в CВС, так что $\sigma I_{\mathrm{OFF}} /\left\langle I_{\mathrm{OFF}}\right\rangle \sim 1$.

Наблюдаемое в эксперименте улучшение отношения $I_{\mathrm{ON}} / I_{\mathrm{OFF}}$, а также уменьшение $V_{\mathrm{SET}}$ и $V_{\mathrm{RESET}}$ при наложении ВЧ синусоидального сигнала на треугольные переключающие импульсы может быть связано, как и в [17], с резонансной активацией дрейфа и диффузии ионов $\mathrm{O}^{2-}$ по кислородным вакансиям в $\mathrm{ZrO}_{2}(\mathrm{Y})$ под действием внешнего переменного электрического поля. Однако из сравнения результатов, полученных в данной работе, с результатами [17] следует, что модулированные ВЧ синусоидальным сигналом переключающие импульсы воздействуют на характеристики РП мемристора с микрометровыми размерами электродов несколько поиному, чем на РП виртуального мемристора, образованного контактом АCM зонда к пленке $\mathrm{ZrO}_{2}(\mathrm{Y})$ на проводящей подложке. Вышеперечисленные различия, в свою очередь, могут быть обусловлены несколькими причинами. Прежде всего следует обратить внимание на различия в размерах и геометрии активной области мемристоров, исследованных в настоящей работе, и виртуальных мемристоров, образованных контактом АСМ зонда к пленке $\mathrm{ZrO}_{2}(\mathrm{Y})$, исследованных в [17]. В первом случае, как уже упоминалось выше, латеральные размеры активной области составляли $\approx 20 \times 20 \mu \mathrm{m}$. Во втором случае размеры виртуального мемристора по порядку величины могут быть приравнены к размерам области контакта острия АСМ зонда к поверхности пленки $\mathrm{ZrO}_{2}(\mathrm{Y}), D_{p} \sim 10 \mathrm{~nm}[20]$. Кроме того, толщина слоя функционального диэлектрика $d$ в мемристорах, исследованных в данной работе $(\approx 40 \mathrm{~nm})$, была в 10 раз выше, чем толщина пленок $\mathrm{ZrO}_{2}(\mathrm{Y})$, исследованных в [17] ( $\approx 4 \mathrm{~nm})$. Несмотря на это, различия в значениях $V_{\text {SET }}$ и $V_{\text {RESET }}$ для обоих случаев были невелики. Данный факт объясняется тем, что при филаментном механизме РП процессы в мемристорах определяются значением напряженности электрического поля в промежутке между одним из электродов и оконечностью филамента (играющего роль виртуального электрода), ближайшей к этому электроду. Толщина этого промежутка составляет $\sim 1 \mathrm{~nm}$, т.е. того же порядка величины, что и толщина диэлектрических пленок, исследованных методом АCM [17]. Таким образом, напряженность электрического поля в области функционального диэлектрика, где происходят основные процессы, ответственные за РП, в обоих случаях приблизительно одинакова. Здесь следует подчеркнуть, что несмотря на то что потенциально под электродами микрометровых размеров может находиться большое количество филаментов, на практике, в процессе электроформинга формируется только один филамент, безотносительно площади электродов мемристорной структуры [27].

Основное отличие режимов исследования РП в настоящей работе и в [17] заключается в том, что в первом случае значения силы тока через мемристор $I$ в ходе электроформинга (ограниченные резистором $R$, см. рис. 1) составляли $\sim 1 \mathrm{~mA}$, тогда как в ходе АСМ измерений [17] значения силы тока через АСМ зонд при электроформинге были $\sim 10 \mathrm{nA}$. Как следствие, диаметр филаментов, формирующихся в мемристорах с микрометровыми контактами при указанных значениях $I$, составляет $\sim 6 \mathrm{~nm}[28]$, что значительно больше диаметра филаментов, формирующихся в пленках $\mathrm{ZrO}_{2}(\mathrm{Y})$ при АCM исследованиях (в пределе - вплоть до атомных размеров). В свою очередь, объем области функционального диэлектрика, в котором напряженность переменной составляющей электрического поля максимальна (т.е. объем области диэлектрической пленки между острием филамента и ближайшим к нему электродом), в первом случае оказывается значительно больше, чем во втором. Необходимо отметить также различия в химическом составе материалов верхних электродов. В мемристорах, исследованных в настоящей работе, на поверхность слоя функционального диэлектрика $\mathrm{ZrO}_{2}(\mathrm{Y})$ наносились химически активные подслои $\mathrm{Zr}$, тогда как в АCM исследованиях [17] АCM зонд (играющий роль подвижного верхнего электрода) был покрыт химически инертной алмазоподобной пленкой.

Однако следует подчеркнуть, что, несмотря на существенные различия в размерах, геометрии и составе материалов электродов мемристоров, исследованных в настоящей работе, и виртуальных мемристоров, исследованных в [17], в целом, общие закономерности влияния наложения ВЧ синусоидального сигнала на переключающие импульсы на параметры РП и их временную стабильность были сходными для обеих систем. Для выяснения деталей механизма влияния дополнительного ВЧ сигнала на параметры и стабильность РП, 
а также причин различий этого влияния в мемристорах с макроскопическими контактами и при исследовании локального РП методом АСМ необходимы дальнейшие экспериментальные и теоретические исследования.

\section{4. Заключение}

Результаты настоящей работы показывают, что наложение на переключающие треугольные импульсы высокочастотного синусоидального сигнала приводит к увеличению отношения значений силы тока через мемристор в низкоомном и высокоомном состояниях, а также к уменьшению напряжения переключения мемристора между указанными состояниями. Обнаруженный эффект может быть связан с резонансной активацией миграции ионов кислорода по кислородным вакансиям под действием внешнего переменного электрического поля. Сравнение результатов настоящей работы с результатами, полученными ранее при исследовании локального РП в тонких пленках $\mathrm{ZrO}_{2}(\mathrm{Y})$ методом АСМ, показывает, что влияние наложения синусоидального сигнала на переключающие импульсы на параметры РП проявляется в обоих случаях сходным образом. В то же время для выяснения деталей механизма влияния дополнительного ВЧ сигнала на параметры и стабильность РП необходимы дальнейшие исследования.

\section{Благодарности}

Исследования выполнены с использованием аппаратных ресурсов центра коллективного пользования Научно-образовательного центра „Физика твердотельных наноструктур“ Нижегородского государственного университета им. Н.И. Лобачевского.

\section{Финансирование работы}

Работа выполнена при финансовой поддержке Российского фонда фундаментальных исследований и Правительства Нижегородской области (проект № 18-42-520059p_a).

\section{Конфликт интересов}

Авторы заявляют, что у них нет конфликта интересов.

\section{Список литературы}

[1] Resistive Switching: From Fundamentals of Nanoionic Redox Processes to Memristive Device Applications / Eds D. Ielmini, R. Waser. Wiley-VCH, Weinheim (2016). 784 p.

[2] R. Waser, M. Aono. Nature Mater. 6, 833 (2007).

[3] I. Riess. J. Electroceram. 39, 61 (2017).

[4] D.B. Strukov, G.S. Snider, D.R. Stewart, R.S. Williams. Nature 453, 80 (2008).

[5] J. Ouyang. Emerging Resistive Switching Memories. Springer, Berlin-Heidelberg (2016). 93 p.
[6] Memristor and Memristive Neural Networks / Ed. A. James. IntechOpen, Rijeka (2018). 314 p.

[7] D. Ielmini. Semicond. Sci. Technol. 31, 063002 (2016).

[8] G.S. Park, Y.B. Kim, S.Y. Park, X.S. Li, S. Heo, M.-J. Lee, M. Chang, J.H. Kwon, M. Kim, U.-I. Chung, R. Dittmann, R. Waser, K. Kim. Nature Commun. 4, 2382 (2013).

[9] J.S. Lee, S. Lee, T.W. Noh. Appl. Phys. Rev. 2, 031303 (2015).

[10] L. Zhu, J. Zhou, Z. Guo, Z. Sun. J. Materiom. 1, 285 (2015).

[11] M. Trapatseli, S. Cortese, A. Serb, A. Khiat, T. Prodromakis. J. Appl. Phys. 121, 184505 (2017).

[12] K.C. Chang, T.C. Chang, T.M. Tsai, R. Zhang, Y.-C. Hung, Y.-E. Syu, Y.-F. Chang, M.-C. Chen, T.-J. Chu, H.-L. Chen, C.-H. Pan, C.-C. Shih, J.-C. Zheng, S.-M. Sze. Nanoscale Res. Lett. 10, 120 (2015).

[13] C. La Torre, K. Fleck, S. Starschich, E. Linn, R. Waser, S. Menzel. Phys. Status Solidi A 213, 316 (2016).

[14] G.A. Patterson, P.I. Fierens, D.F. Grosz. Appl. Phys. Lett. 103, 74102 (2013).

[15] V. Ntinas, A. Rubio, G.Ch. Sirakoulis, S.D. Cotofana. In: Proc. 2019 IEEE Int. Symp. Circuits and Systems. Sapporo, Jpn (May 16-20, 2019). P. 1.

[16] В.С. Анищенко, А.Б. Нейман, Ф. Мосс, Л. ШиманскийГайер. УФН 169, 7 (1999).

[17] Д.О. Филатов, М.Н. Коряжкина, Д.А. Антонов, И.Н. Антонов, Д.А. Лискин, М.А. Рябова, О.Н. Горшков. ЖТФ 89, 1669 (2019).

[18] F. Pan, J. Jang, V. Subramanian. In: Proc. IEEE $70^{\text {th }}$ Dev. Res. Conf. University Park, PA, USA (June 18-20, 2012). P. 217.

[19] S. Ambrogio, B. Magyari-Köpe, N. Onofrio, M.M. Islam, D. Duncan, Y. Nishi, A. Strachan. J. Electroceram. 39, 39 (2017).

[20] D.O. Filatov, D.A. Antonov, O.N. Gorshkov, A.P. Kasatkin, D.A. Pavlov, V.N. Trushin, I.A. Antonov, M.E. Shenina. In: Atomic Force Microscopy (AFM): Principles, Modes of Operation and Limitations / Ed. H. Yang. Nova Science, N.Y. (2014). P. 335.

[21] J.Y. Seok, S.J. Song, J.H. Yoon, K.J. Yoon, T.H. Perk, D.E. Kwon, H. Lim, G.H. Kim, D.S. Jeong, C.S. Hwang. Adv. Funct. Mater. 24, 5316 (2014).

[22] M. Lanza. Materials 7, 2155 (2014).

[23] O.N. Gorshkov, A.N. Mikhaylov, A.P. Kasatkin, S.V. Tikhov. J. Phys., Conf. Ser. 741, 012174 (2016).

[24] S.V. Tikhov, O.N. Gorshkov, I.N. Antonov, A.E. Morozov, M.N. Koryazhkina, D.O. Filatov. Adv. Cond. Matter Phys. 2018. Article ID 2028491.

[25] A.V. Yakimov, D.O. Filatov, O.N. Gorshkov, D.A. Antonov, D.A. Liskin, I.N. Antonov, A.V. Belyakov, A.V. Klyuev, A. Carollo, B. Spagnolo. Appl. Phys. Lett. 114, 253506 (2019).

[26] В.Г. Заводинский. ФТТ 46, 441 (2004).

[27] R. Waser, R. Dittmann, G. Staikov, K. Szot. ADV. Mater. 21, 2632 (2009).

[28] Е.В. Окулич, М.Н. Коряжкина, Д.С. Королев, А.И. Белов, М.Е. Шенина, А.Н. Михайлов, Д.И. Тетельбаум, И.Н. Антонов, Ю.А. Дудин. Письма в ЖТФ 45, 14, 3 (2019).

Редактор Ю.Э. Китаев 